\title{
Formación de películas anódicas en una aleación Al-W: Migración de fósforo
}

\author{
Sandra Judith García Vergara \\ PhD en Ciencia e Ingeniería de la Corrosión, \\ Universidad de Manchester \\ Docente Tiempo Completo, Investigadora, Escuela de Ingeniería \\ Metalúrgica, Universidad Industrial de Santander UIS \\ Bucaramanga, Colombia \\ Fernando Viejo Abrante \\ PhD Ciencia y Tecnología de Materiales, \\ Universidad Complutense de Madrid \\ Docente Tiempo Completo, Investigador, Escuela de Ingeniería \\ Química, Universidad Industrial de Santander UIS \\ Bucaramanga, Colombia \\ fviejo@uis.edu.co
}

\author{
Ana Emilse Coy Echeverría \\ $\mathrm{PhD}$ en Ciencia y Tecnología de Materiales, \\ Universidad Complutense de Madrid \\ Docente Tiempo Completo, Investigadora, \\ Escuela de Ingeniería Metalúrgica y Ciencia de Materiales, \\ Universidad Industrial de Santander UIS \\ Bucaramanga, Colombia \\ anaecoy@uis.edu.co \\ George E. Thompson \\ PhD en Desarrollo de Nuevas Aleaciones Ligeras \\ Profesor, Universidad de Manchester \\ Manchester, Inglaterra \\ george.thompson@ manchester.ac.uk
}

\author{
Peter Skeldon \\ Profesor, Universidad de Manchester \\ Manchester, Inglaterra \\ p.skeldon@manchester.ac.uk
}

\begin{abstract}
Resumen- El presente trabajo investiga la incorporación y migración de especies de fósforo en las películas anódicas y su interacción con las especies de wolframio incorporadas a las películas anódicas desde el sustrato metálico. El estudio usa películas de alúmina, formadas sobre una aleación Al-15at.\%W depositada catódicamente, en soluciones que contienen fosfatos. La morfología y la composición de las películas se determinaron por microscopia electrónica de barrido (SEM) y espectroscopia de emisión óptica (GDOES). Se observó que las películas están constituidas o por una región externa rica en wolframio y una región interna relativamente libre de wolframio, o solamente por una región rica en wolframio. Las especies de fósforo migran hacia dentro de la película más despacio en las películas que contienen wolframio que en las que no contienen wolframio. En contraste, la migración de las especies de wolframio hacia la solución no se ve afectada por la presencia de fósforo.
\end{abstract}

Palabras clave- Aluminio, wolframio, películas anódicas, GDOES.

Abstract-The interaction between phosphorus species and tungsten species, both incorporated within anodic alumina is investigated. The study used anodic films, formed on a sputtering-deposited Al-15at.\%W alloy in phosphate containing electrolytes. The morphology and composition of the anodic films were determined by scanning electron microscopy (SEM) and glow discharge ion emission spectroscopy (GDOES). The films consisted of either an outer tungsten-containing region and an inner tungsten-free region, or a tungsten-containing region only. Phosphorus species were shown to migrate inward in the tungsten-containing alumina more slowly than in the tungsten-free alumina. In contrast, the out- ward migration of tungsten species was relatively unaffected by the presence of phosphorus species.

Keywords-Aluminium, tungsten, anodic film, GDOES.

\section{INTRODUCCIÓN}

Las películas de alúmina se usan ampliamente en la protección y funcionalización de las aleaciones de aluminio para aplicaciones tanto en la industria electrónica, como automotriz y aeroespacial, hasta litográfica, por sólo mencionar algunas [1]. El crecimiento de películas anódicas normalmente conlleva a la incorporación de especies derivadas de los aniones que contiene la solución de anodizado a la capa externa de la película [2]. La distribución de estas especies incorporadas está directamente relacionada con sus velocidades de migración relativas a las de los iones del metal o a las de los iones de oxígeno [3]. Se ha observado que las especies que se incorporan son aquellas que pueden migrar desde la solución hacia el interior de la película de alúmina, lo cual es una consecuencia directa del hecho que la película de alúmina crece debido a la migración de iones de oxígeno provenientes de la solución de anodizado [4]. La incorporación de especies se ha observado cuando el anodizado se lleva a cabo en ácidos oxálico, fosfórico o sulfúrico [5], en contraste cuando el anodizado se realiza en ácido crómico se ha 
observado una incorporación mínima de especies de cromo [6]. La eficiencia del proceso de anodizado, es decir la relación entre el espesor de la película de alúmina formada respecto al espesor del aluminio consumido, y la morfología de los poros se ven afectados por la presencia de dichas especies incorporadas desde el electrolito [7]. El mecanismo de formación de las películas anódicas ha sido recientemente estudiado mediante capas de trazador de una aleación AI-W en un sustrato de aluminio, las cuales son luego incorporadas a la película anódica durante el proceso de anodizado [7],[8] [9]. Los iones de $\mathrm{W}^{6+}$ migran lentamente hacia la solución a través de la capa barrera de las películas porosas. En las películas anódicas con el trazador de wolframio, éste nunca alcanza la base del poro, y muestran un comportamiento opuesto al observado cuando las películas anódicas no contienen ninguna especie incorporada desde la solución. Esto significa que la presencia de estas especies incorporadas podría influenciar el transporte de los iones de wolframio en la capa barrera. En el presente trabajo se estudia el crecimiento de películas anódicas en una aleación Al-W. El anodizado se llevó a cabo en soluciones que contienen especies de fósforo, para que luego sean incorporadas a las películas anódicas y así poder establecer si existe alguna interacción de las especies de fósforo incorporadas desde la solución con las especies de wolframio incorporadas desde el sustrato metálico.

\section{DESCRIPCIÓN EXPERIMENTAL}

Una aleación con composición promedio Al15at.\%W se depositó por magnetrón sputtering mediante un sistema de vacío Products Corp. Se usaron como targets para la deposición discos de aluminio y de wolframio de $99.99 \%$ de pureza. La deposición se llevó a cabo de manera que se produjeron multicapas finas en la aleación las cuales son alternadamente ricas y pobres en wolframio relativo a la composición promedio de la aleación. La aleación se depositó sobre sustratos electropulidos de aluminio de alta pureza con dimensiones de $1.5 \times 3.0 \mathrm{~cm}$. El espesor total de de aleación depositada fue de $495 \mathrm{~nm}$, el cual contiene 27 capas ricas en wolframio. Con un área de trabajo de $2 \mathrm{~cm}^{2}$, las muestras fueron anodizadas a $5 \mathrm{~mA} /$ $\mathrm{cm}^{2}$ hasta $140 \mathrm{~V}$ en una solución $0.1 \mathrm{M}$ de fosfato de sodio $\left(\mathrm{Na}_{2} \mathrm{HPO}_{4}\right)$ a $293 \mathrm{~K}$. Para el anodizado se empleó una celda de dos electrodos con un electrodo auxiliar de aluminio. La solución fue agitada durante el anodizado. Algunas muestras fueron también anodizadas a $5 \mathrm{~mA} / \mathrm{cm}^{2}$ hasta $150 \mathrm{~V}$ en un solución $0.1 \mathrm{M}$ de pentaborato de amonio $(\mathrm{NH}-$ ${ }_{4} \mathrm{~B}_{5} \mathrm{O}_{8} .4 \mathrm{H}_{2} \mathrm{O}$ ) a $293 \mathrm{~K}$, o hasta $212 \mathrm{~V}$ en una solución $0.4 \mathrm{M}$ de ácido fosfórico a 293K. Además se anodizaron muestras de aluminio de alta pureza eletropulido hasta 140 en la solución de fosfato de sodio, para producir películas anódicas libres de wolframio que sirvieran como referencia. Todas las muestras fueron lavadas con agua desionizada luego del anodizado y secadas con aire frío.

Para los análisis por espectroscopia de emisión óptica por descarga luminiscente (rf-GDOES) se usó un equipo GD-Profiler 2 (Horiba Jobin Yvon), operado a una frecuencia de $13.56 \mathrm{MHz}$. Como ánodo se empleó un disco de cobre de $4 \mathrm{~mm}$ de diámetro y se utilizó gas argón. La respuesta de la emisión correspondiente a los elementos excitados durante la descarga se detectó con un policromatógrafo de longitud focal $500 \mathrm{~mm}$ con 30 ventanas ópticas. Los perfiles de concentración se llevaron a cabo a $700 \mathrm{~Pa}$ y $35 \mathrm{~W}$, con un tiempo de adquisición de los datos de $0.005 \mathrm{~s}$. Las líneas de emisión (nm) usadas para el análisis fueron 396.15 para aluminio, 249.678 para boro, 130.21 para oxígeno, 178.28 para fósforo y 429.46 para wolframio. El diámetro del área de análisis fue $4 \mathrm{~mm}$. Varios análisis de GDOES se hicieron en cada muestra para asegurar la reproducibilidad de los resultados.

Las muestras anodizadas fueron examinadas por microscopia electrónica de barrido (SEM) con un equipo Zeiss Ultra 55. El voltaje de aceleración fue de $1.5 \mathrm{kV}$. Para este análisis se emplearon secciones transversales de las muestras preparadas por ultramicrotomía mediante un cuchillo de vidrio como elemento de corte seguido por cortes con un cuchillo de diamante.

\section{RESULTADOS Y DISCUSIÓN}

\section{A. Curvas voltaje-tiempo}

La Fig. 1 muestra la variación del voltaje con el tiempo de anodizado para la aleación Al-15at.\% en las soluciones que contienen fosfatos. Para el anodizado en fosfato de sodio, el voltaje aumenta linealmente con el tiempo hasta alcanzar un voltaje de $140 \mathrm{~V}$, a una razón de $2.5 \mathrm{~V} / \mathrm{s}$. Lo cual sugie- 
re que la película barrera está siendo formada con una eficiencia del 100\%. Un comportamiento similar se observa para el anodizado de la aleación en pentaborato de amonio. En contraste el comportamiento difiere cuando el anodizado se lleva a cabo en ácido fosfórico. Se observa una región lineal en la cual el voltaje aumenta linealmente con el tiempo, a una razón de $1.6 \mathrm{~V} / \mathrm{s}$, seguida por otra región en la que el voltaje oscila, hasta alcanzar un valor de $212 \mathrm{~V}$. La pendiente de la región lineal es menor que la observada para las otras dos soluciones de anodizado, sugiere que hay una pérdida de especies de aluminio o wolframio desde la película hacia la solución de anodizado. En este caso se espera que la morfología de la película anódica comprenda la formación de una capa porosa externa, como luego se demostrará con el análisis por SEM.

FIG. 1. CURVA VOLTAJE TIEMPO PARA LA ALEACIÓN AL-15AT.\%W ANODIZADA A 5 MA/CM² EN A) SOLUCIÓN 0.1 M DE FOSFATO DE SODIO HASTA 141 V, B) SOLUCIÓN O.1 M DE PENTABORATO DE AMONIO HASTA 152 V Y C) SOLUCIÓN O.4 M DE ÁCIDO FOSFÓRICO HASTA $212 \mathrm{~V}$

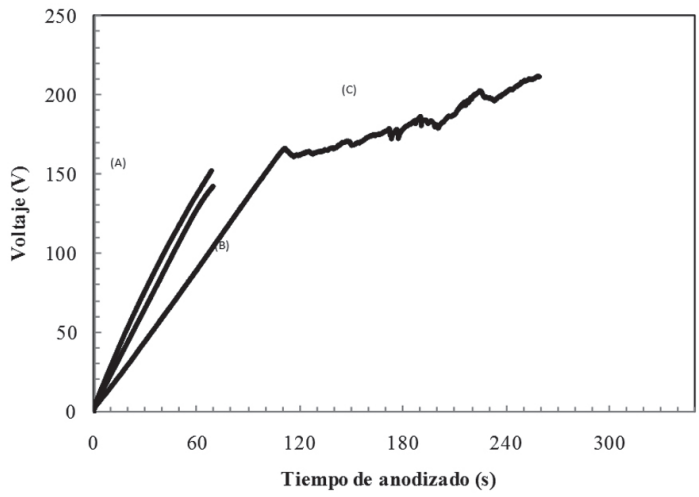

\section{B. Análisis de las películas anódicas por rf- GDOES}

La Fig. 2.a) muestra los resultados del análisis por rf-GDOES, la dependencia de la emisión óptica del oxígeno, aluminio y fósforo, en unidades arbitrarias, sobre el tiempo de remoción (sputtering), para una muestra de aluminio anodizada hasta $140 \mathrm{~V}$ en la solución de fosfato de sodio. La señal correspondiente al oxígeno disminuye a niveles muy bajos luego de 10 s, lo cual indica que la película anódica ha sido removida en su totalidad y que se alcanzó la interface óxido/aluminio. Al mismo tiempo la señal correspondiente al aluminio, la cual fue relativamente constante durante la remoción de la película anódica, aumenta considerablemente tan pronto como comienza la remoción del sustrato de aluminio. La señal para el fósforo cae a niveles muy bajos antes de que la señal de oxígeno se reduzca lo cual indica que el fósforo está presente en la región exterior de la película anódica y que las regiones interiores de la película están libres de fósforo. Las ondulaciones observadas en la señal del fósforo dentro de las películas anódicas pueden deberse a interferencias ópticas. El tiempo total requerido para remover todo el espesor de óxido fue $8.5 \mathrm{~s}$. Si la velocidad de remoción de la película anódica es constante, la relación de los tiempos corresponde a la relación del espesor de la región del óxido que contiene fósforo respecto al espesor total del óxido. Mediciones de la distribución del fósforo en la alúmina anódica mediante análisis químico de cortes transversales de la película [10], [11], [12] y microscopia electrónica de barrido [13], indican que las especies de fósforo están presentes desde la superficie de la película hasta profundidades relativas de 0.7-0.8 del espesor total y la parte restante está compuesta de alúmina libre de fósforo. La buena correspondencia de estos resultados con los reportados en el presente estudio por rf-GDOES, indican que las velocidades de remoción de las regiones del óxido que contienen o no fósforo son similares.

El análisis por rf-GDOES de la aleación Al15at.\%W anodizada hasta $141 \mathrm{~V}$ en fosfato de sodio se presenta en la Fig. 2b). La película anódica contiene aluminio, oxígeno, fósforo y wolframio. La señal para el wolframio comienza más tarde que las señales para el oxígeno y el aluminio, que indica que el wolframio está presente en las regiones más internas de la película anódica. El momento en el que se ha removido todo el óxido, es decir, se alcanza la interface óxido/aleación, se evidencia por una disminución en la señal del oxígeno, acompañada a la vez por un aumento en las señales del aluminio y del wolframio. En este punto se comienza a remover la aleación. La relación de los tiempos necesarios para remover toda la región del óxido que contiene wolframio respecto al espesor total del óxido es de 0.61. El fósforo está presente en las regiones exteriores de la película, y su señal sobrepasa parte de la señal del wolframio. Al considerar que la presencia 
de fósforo no afecta la velocidad de remoción de la película anódica en aluminio puro y que, además, es constante en la región que contiene wolframio, se calcula que el fósforo está presente en cerca de 0.34 de la región que contiene wolframio.

La relación de los tiempos necesarios para remover toda la región que contiene fósforo y todo el espesor de la película anódica es de 0.60. La señal para el aluminio disminuye en el momento en que pasa la frontera entre las regiones que contienen y no contienen wolframio, lo cual puede ser debido a la presencia de wolframio en la región interna. Debido a que se aumenta la velocidad de remoción en la región que contiene wolframio, así como un pequeño incremento en el voltaje de anodizado, remover todo el espesor de la película toma $6.2 \mathrm{~s}$, el cual es mucho menos que el necesario para remover una película anódica formada sobre aluminio, el cual es 8.5s.

La Fig. 2c muestra los perfiles de concentración obtenidos por rf-GDOES para la aleación $\mathrm{Al} / 15 . \% \mathrm{~W}$ anodizada hasta $152 \mathrm{~V}$ en pentaborato de amonio. La película contiene una región exterior libre de wolframio y una región interna que contiene wolframio, la relación entre los tiempos de remoción necesarios para quitar toda la región interna y toda la película, fue de 0.61. Un valor muy similar se obtuvo para la película formada en la solución de fosfato de sodio.

FIG. 2. PERFILES DE CONCENTRACIÓN OBTENIDOS POR GDOES PARA (A) ALUMINIO ANODIZADO HASTA 141V A 5MA/CM² EN UNA SOLUCIÓN 0.1M FOSFATO DE SODIO; (B) ALEACIÓN AL-15AT.\%W ANODIZADA HASTA 141V A 5MA/CM ${ }^{2}$ EN UNA SOLUCIÓN DE FOSFATO DE SODIO; (C) ALEACIÓN AL-15AT.\%W ANODIZADA HASTA 152V A 5MA/CM² EN UNA SOLUCIÓN 0.1M PENTABORATO DE AMONIO; Y (D) ALEACIÓN AL-15AT.\%W ANODIZADA HASTA 165V A 5MA/CM² EN UNA SOLUCIÓN O.4 M ÁCIDO FOSFÓRICO

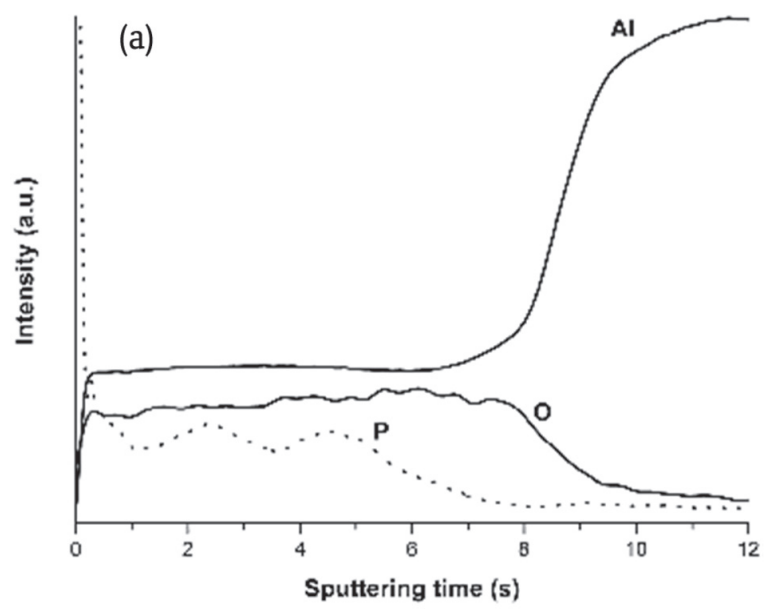

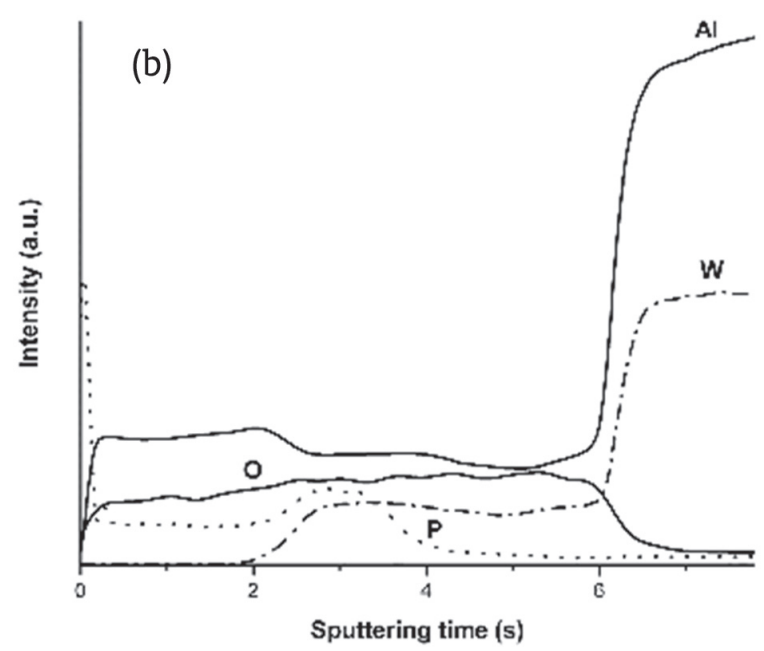
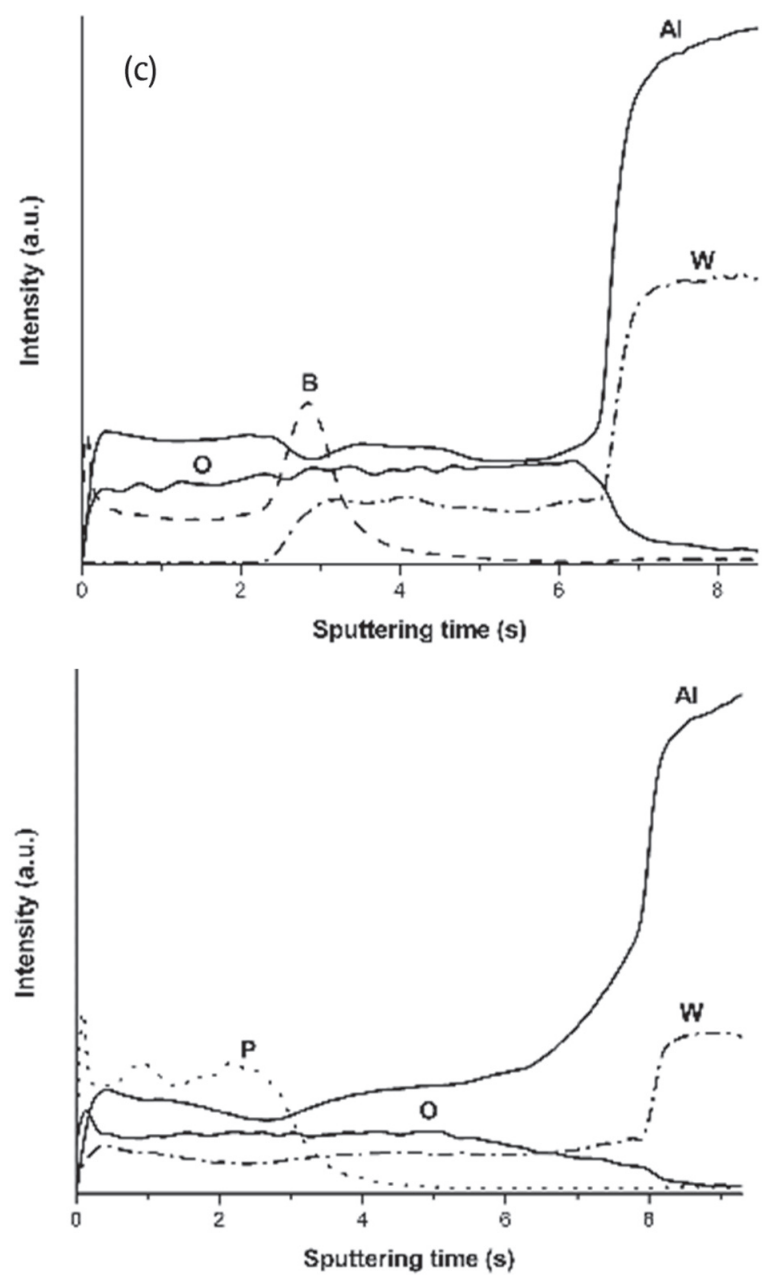

Los perfiles de concentración de rf-GDOES para la aleación $\mathrm{Al}-15$ at.\%W anodizada hasta $165 V$ en ácido fosfórico se presentan en la Fig. 2d. Los perfiles muestran que las películas contienen wolframio en todo su espesor. La señal del oxígeno es menos pronunciada en la interface óxido/ 
aleación comparada con las muestras anteriores. Esto se debe a que la película no es uniforme. La película está formada por una región tipo barrera de un espesor uniforme y otra región externa con poros finos, como se observa en las micrografías obtenidas por SEM. Hay presencia de fósforo en la región exterior de la película, la cual corresponde a cerca de 0.43 del espesor total.

\section{Análisis de las películas anódicas por SEM}

El boro es incorporado en la región externa, la cual está a su vez libre de wolframio. Sin embargo, se observa un pico en la señal del boro, localizado cerca de la frontera entre las regiones que contienen o no wolframio. La relación de los tiempos de remoción hasta el pico de boro y de toda la película es 0.42. También hay otro pequeño pico en la señal del boro localizado en la superficie de la película, el cual es debido a las especies de boro que son absorbidas desde la solución. El tiempo necesario para remover toda la película fue $6.6 \mathrm{~s}$, el cual es $0.4 \mathrm{~s}$ mayor que el tiempo necesario para remover toda la película formada en la solución de fosfato de sodio. La variación puede deberse a las diferencias entre el voltaje de anodizado y la composición de las películas.

La Fig. 3a muestra una micrografía de la sección transversal por SEM, de la aleación AI15at.\%W anodizada en fosfato de sodio. La película anódica tiene un espesor uniforme de 168nm. Sin embargo la superficie de la película y la interface óxido/aleación aparecen onduladas. La relación entre el espesor de la película y el voltaje de formación es de $1.19 \mathrm{~nm} / \mathrm{V}$, valores similares han sido reportados en películas libres de wolframio [14].

FIG. 3. MICROGRAFÍAS ELECTRÓNICAS DE BARRIDO (ELECTRONES RETRODISPERSADOS) DE LA ALEACIÓN AL-15AT.\%W ANODIZADA A 5MA/CM ${ }^{2}$ EN A) SOLUCIÓN O.1 M DE FOSFATO DE SODIO HASTA 141 V, (B) SOLUCIÓN O.1 M DE PENTABORATO DE AMONIO HASTA $152 \mathrm{~V}$ Y (C) SOLUCIÓN O.4M

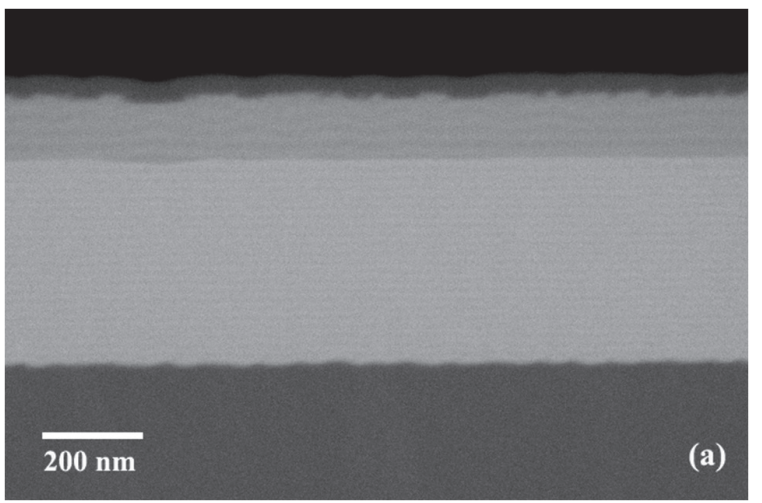

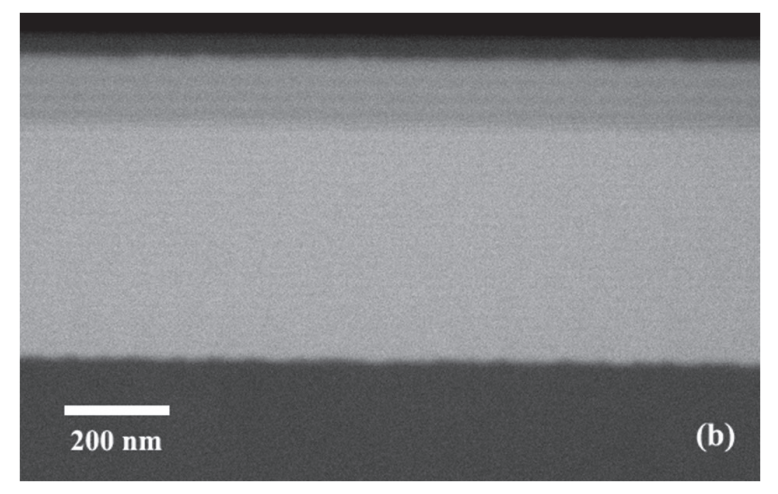

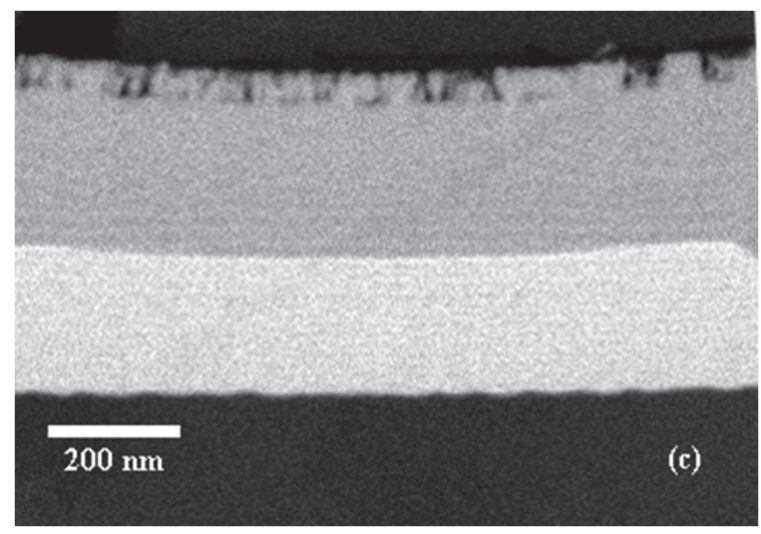

DE ÁCIDO FOSFÓRICO HASTA 212V

Se distinguen capas brillantes ricas en wolframio, las cuales están alternadas con capas más oscuras, menos ricas en wolframio. La película anódica claramente contiene una región exterior, oscura, libre de wolframio y una región interna más brillante, en la que se observan capas ricas en wolframio. La película contiene cinco capas, las cuales aparecen más espaciadas unas de otras que las capas en la aleación sin anodizar, con una relación de 1.57. Los espesores promedio de la región interna que contiene wolframio y de la interna que no contiene son 128 y 40nm, y la relación entre el espesor de la región externa y el espesor total de la película es de 0.24.Los espesores de las películas determinados por SEM y por rf-GDOES pueden usarse para calcular las velocidades promedio de remoción (sputtering) de las regiones que contienen y no contienen wolframio. Así la región libre de wolframio, con un espesor de 40nm, es removida en $2.4 \mathrm{~s}$, dando una velocidad de remoción de $17 \mathrm{~nm} / \mathrm{s}$. La región que contiene wolframio con un espesor de $128 \mathrm{~nm}$, es removida en $3.8 \mathrm{~s}$, dando una velocidad de remoción de $34 \mathrm{~nm} / \mathrm{s}$, la cual es casi el doble de la velocidad necesaria para remover la región que no tiene wolframio. Usando estos 
valores puede calcularse que el fósforo está presente en 0.51 de la parte exterior de la película.

La micrografía por SEM de las secciones transversales de la película anódica formada en pentaborato de amonio se presenta en la Fig. 3b. La película tiene un espesor total de $177 \mathrm{~nm}$, el cual comprende $45 \mathrm{~nm}$ libres de wolframio localizados en la región exterior y $132 \mathrm{~nm}$ que contienen wolframio en la región interior. La relación entre el espesor de la capa externa respecto al espesor total de la película es 0.25 , muy similar al valor obtenido para la película formada en fosfato de sodio. Sin embargo, la película es más homogénea y la superficie de la película, así como la interface óxido/ aleación son más regulares. Las bandas ricas en wolframio también aparecen más rectas una vez incorporadas a la película de óxido. La relación de formación de la película es de 1.16nm/V. La película contiene cinco bandas ricas en wolframio, y la relación de separación entre las bandas es de $1.60 \mathrm{~nm}$. Similarmente puede calcularse las velocidades para remover las regiones con o sin wolframio como 18 y $33 \mathrm{~nm} / \mathrm{s}$, los valores son similares a los calculados para las películas formadas en fosfato de sodio.

La Fig. 3c muestra una micrografía de SEM de la película anódica formada en acido fosfórico. La película está compuesta de una capa barrera de $228 \mathrm{~nm}$ de espesor y una capa externa con poros finos y un espesor de 48nm. La región porosa representa un 0.17 del espesor total de la película. La relación de formación es $1.08 \mathrm{~nm} / \mathrm{V}$. Cerca de catorce bandas ricas en wolframio han sido oxidadas durante la formación de la película anódica. Las bandas de wolframio pueden verse claramente en la región no porosa, pero son menos evidentes en la región porosa. Las bandas aparecen onduladas. La relación entre el espaciamiento de las bandas en la película y en la aleación es de 1.62, valor similar al observado con las películas formadas en fosfato de sodio y en pentaborato de amonio. Con el espesor de la película determinado por SEM y el tiempo de remoción determinado por rf-GDOES, la velocidad de remoción puede calcularse y es de $31 \mathrm{~nm} / \mathrm{s}$.

\section{Interacción entre fósforo y wolframio en las películas anódicas}

El anodizado de la aleación Al-15at.\%W en fosfato de sodio produce películas anódicas, en las cuales el wolframio y el fósforo no se encuentran regularmente distribuidos. Las especies de wolframio son incorporadas en la interface aleación/ óxido, como unidades de $\mathrm{WO}_{3}$ [15]. Los iones de wolframio migran dentro de la película a una velocidad menor que los iones de aluminio, razón por la cual la parte exterior de la película no contiene wolframio. En contraste las especies de fósforo son incorporadas en la interface óxido/solución y migran hacia dentro a una velocidad menor que los iones de oxígeno [3]. La formación de las películas anódicas es el resultado de la migración iónica del aluminio presente en la aleación y del oxígeno presente en la solución de anodizado. Lo cual conlleva a que las películas crezcan con una eficiencia alta. Sin embargo en el caso del anodizado en acido fosfórico, la película anódica se forma por la migración de solamente iones de oxígeno, ya que los iones de aluminio se pierden en la solución de anodizado [16].

Los presentes resultados sugieren que las especies de fósforo migran más lentamente a través de la película debido a la presencia de wolframio. La razón por la cual la velocidad de migración disminuye puede estar relacionada con la modificación del enlace de las especies de fósforo en la alúmina que contiene wolframio y la influencia de las especies de wolframio en la fuerza de los campos eléctricos locales. La reducción en la migración de las especies de fósforo relativa a las especies de oxígeno conlleva a un incremento en la concentración de iones de fósforo en la región de la película rica en wolframio respecto a la región que no contiene wolframio.

Comparando las películas formadas en fosfato de sodio con las formadas en pentaborato de amonio, es evidente que la región interna de ambas películas contiene especies de wolframio, y la región externa contiene fósforo o boro respectivamente. Los resultados indican que la presencia de fósforo no afecta la velocidad de migración de las especies de wolframio.

Un comportamiento diferente es observado con las películas anódicas formadas en ácido fosfórico. Estas películas no muestran una región externa libre de wolframio. El fósforo está presente en las regiones externas de la película que también contienen wolframio. La porción del óxido que contiene fósforo es mayor que en las películas formadas en fosfato de sodio. Las películas se for- 
man a una eficiencia menor y presentan una región externa porosa la cual se debe a la formación inicial de caminos de penetración en la superficie de la película, seguida por una disolución de la película asistida por el campo eléctrico.

\section{CONCLUSIONES}

El anodizado a alta eficiencia de una aleación Al-15at.\%W a $5 \mathrm{~mA} / \mathrm{cm}^{2}$ en $0.1 \mathrm{M}$ de fosfato de sodio, lleva a la formación de una película compuesta de una región externa de alúmina libre de wolframio y una región interna de alúmina que contiene wolframio, con especies de fósforo incorporadas en ambas regiones de la película. El fósforo está distribuido en una región comprendida entre la superficie de la película y la mitad del espesor total de la película.

El anodizado de la aleación en 0.4M de ácido fosfórico causa la formación de una película que contiene especies de wolframio en todo su espesor, sin una región de alúmina libre de wolframio. Se observa una capa porosa externa en la superficie de la película. Especies de fósforo son incorporadas en la región externa de la película y migran hacia dentro con una velocidad menor que las especies de oxígeno. Sin embargo hay una región libre de fósforo en la interface óxido/ aleación. La velocidad de migración del fósforo se ve significantemente reducida por la presencia de wolframio en las películas. Sin embargo, la presencia de fósforo no parece afectar la velocidad de migración del wolframio.

Especies de boro son incorporadas en la región exterior de las películas formadas en pentaborato de amonio. Sin embargo, la cantidad de especies de boro presentes en la región de alúmina que contiene wolframio es mínima. El boro se acumula en la frontera con la región que contiene wolframio.

\section{AGRADECIMIENTOS}

Los autores desean expresar su agradecimiento al Consejo de Investigaciones en Ingeniería y Ciencias Físicas (EPSRC: Portfolio Award) del Reino Unido y a la Vicerrectoría de Investigación y Extensión de la Universidad Industrial de Santander (Proyecto: Formación de películas nanoporosas en aluminio, Código 5440) en Colombia, por la financiación de esta investigación.

\section{REFERENCIAS}

[1] P. G. Sheasby, R. Pinner, The surface treatment and finishing of aluminium and its alloys, ASM International, Finishing Publications Ltd, United Sates, 2001

[2] R.B. Mason, J. Electrochem. Soc, 1955, 102, 671

[3] G. C. Wood, P. Skeldon, G. E. Thompson, K. Shimizu, J. Electrochem. Soc. 1996, 143, 74

[4] C. Cherki, J. Siejka, J. Electrochem. Soc, 1973, 120,784

[5] G. E. Thompson, R. C. Furneaux, G. C. Wood, Corros. Sci. 1978, 18, 481

[6] G. E. Thompson, G. C. Wood, R. Hutchings, Trans. Inst. Met. Finish, 1980, 58, 21

[7] S. J. Garcia-Vergara, P. Skeldon, G.E. Thompson, H. Habazaki, Electrochim. Acta, 2006 , 52, 681

[8] S. J. Garcia-Vergara, P. Skeldon, G.E. Thompson, H. Habazaki, Surf. Interface Anal. 2007, 31, 860

[9] F. Zhou, D.J. Leclere,. S.J. Garcia-Vergara, T. Hashimoto,. I.S. Molchan. H. Habazaki,. P. Skeldon, G.E. Thompson, J. Electrochem. Soc., 2010, 157, C437

[10] H. Takahashi, K. Fujimoto, M. Nagayama, J. Electrochem. Soc. $1988,135,1349$

[11] J. J. Randall, Jr, W. J. Bernard, Electrochim. Acta 1975, 20, 653.

[12] S.J. García-Vergara, D. Le Clere, T. Hashimoto, H. Habazaki, P. Skeldon, G.E. Thompson, Electrochim. Acta 2009, 54,6403

[13] G. E. Thompson, G. C. Wood, K. Shimizu, Electrochim. Acta 1981, 26,951

[14] G. E. Thompson, G. C. Wood, K. Shimizu, Electrochim. Acta 1981, 26, 951

[15] H. Habazaki, K. Shimizu, P. Skeldon, G. E. Thompson, G. C. Wood, J. Electrochem. Soc. 1996, 143, 2465

[16] M. Curioni, E.V. Koroleva, P. Skeldon, G.E. Thompson, Electrochem. Acta, 2010, 55, 7044. 